\title{
Diagnosis of cervico facial lymphadenopathy by color doppler ultrasound
}

\author{
Gayathri. $\mathbf{R}^{1, *}$, G. Maragathavalli², T.N. Umamaheswari ${ }^{3}$, Prabhu. $\mathbf{K}^{4}$ \\ ${ }^{1,4}$ Reader, ${ }^{2}$ Professor and HOD, ${ }^{3}$ Professor, ${ }^{1-3}$ Dept. of Oral Medicine and Radiology, ${ }^{4}$ Dept. of Prosthodontics, ${ }^{\mathbf{1 , 4}}$ Adhiparasakthi \\ Dental College, Tamil Nadu, ${ }^{2,3}$ Saveetha Dental College, Tamil Nadu, India \\ *Corresponding Author: \\ Email: gayathrisuren19@gmail.com
}

\begin{abstract}
Aim: The aim of the research is to establish diagnosis of diseases in cervico facial lymphadenopathy by colour Doppler ultrasound.

The purpose of the present study is to study the clinical aspects of lymph node and the changes in node in colour doppler ultrasonography.

Materials and Methods: Patients were clinically examined by bimanual palpation for cervico facial lymphadenopathy and their colour doppler ultrasound findings were recorded. Biopsy was done to confirm ultrasound findings.

Results: The internal architecture of the node, the pattern of colour doppler flow, vascular resistance were assessed in various inflammatory and malignant lymph nodes.

Conclusion: The utility of colour doppler ultrasound is really beneficial in establishing the regional lymph node enlargement as inflammatory and metastatic node.
\end{abstract}

Keywords: Lymphnode, Colour doppler, Ultrasound, Reactive nodes, Necrosis, Blood flow.

\section{Introduction}

Oral squamous cell carcinoma is a malignant disease that develops from dysplastic epithelium of oral mucosa. The important characteristic feature of oral squamous cell carcinoma is that the lesion has got the abilty to metastasize in the regional area and remote parts of the body through lymphatic and haematogenous route. Most oral carcinoma metastasize into different lymph nodes of neck region.

Clinically differentiating inflammatory lymph node from carcinomatous lymph nodes is by the presence of stony hard consistency of lymph nodes and their fixation to deeper structures in metastatic lymph nodes whereas inflammatory lymph nodes are firm, tender and movable, and not fixed.

The modern treatment planning in oral carcinoma rules on the staging based on imaging techniques to detect regional and distant metastasis. Thus, the study of lymph node involvement is not only useful for diagnosis of oral carcinoma but also for treatment planning.

Although sophisticated imaging techniques are available to study the involvement of lymph nodes in oral carcinoma, colour doppler ultrasound is a simple, quick, non-invasive, cost effective technique that uses non ionising radiation when compared to the other imaging modalities and is good to assess the vascularity of the lesion or lymph node in carcinoma. ${ }^{1-4}$

The purpose of the study is to co-relate the clinical, and histopathological observation with the finding of colour doppler ultrasonography in carcinomatous as well as inflammatory lymph nodes.

\section{Materials and Methods}

This study was done for partial fulfillment for postgraduation course in saveetha dental college.

Subjects: A total number of 15 patients who were having cervicofacial lymph node enlargement due to oral squamous cell carcinoma and non-cancerous lesions were taken for the color doppler sonography. Age range of the males and females were between 13 and 70 years.

Group 1: 6 cases of oral Squamous cell carcinoma (3 males and 3 females).

Group 2: Non-cancerous lesions ( 9 cases)

2 cases of tuberculous lymphadenitis

1 case of lymphoma

6 cases of inflammatory conditions- alveolar, periodontal abscess, and pericoronitis.

\section{Armamentarium for Biopsy and FNAC}

Bp blade No.15 with handle

Mouth Mirror

Probe

Scissors

Tweezers

Kidney Tray

Syringe and needle

Xylocaine

Chlorhexidine

Tissue forceps

Formalin

Plastic vial

Cotton and gauze

Suture material

Suture needle

Glass Slide

Radiographs of the Jaw

Intra Oral Peri Apical Radiographs 
Occlusal Radiographs

Orthopantomograms

Lateral Oblique

Chest X-ray

Blood Investigations

Erythrocyte sedimentation Rates

Mantoux test

Ultrasonogram

GE voluson 730 pro with Linear Transducer- $7.5 \mathrm{Mh}$

\section{Methodology}

Clinical Examination of Patients with oral Cancer

Lesions: The various clinical varieties of oral carcinoma presented in the form of extra-oral swelling, ulcer, ulcero-proliferative growth and fissured growth with lymph node involvement in the cervico-facial region that were hard or firm, fixed or movable, tender or non-tender on palpation.

Systemic Evaluation of Patients by General Physician: Medical examination was done by the physician for all the patients and investigations like chest radiograph and blood investigations were carried out for the patients with suspected tuberculosis patient, and for the patients with inflammatory nodes.

Examination of Lymph Node- Clinical Methodology: The patients is seated upright with the head slightly tilted downwards in the dental chair and the patients were most effectively examined from behind. Palpation of the cervical lymph nodes was done by bi-manual palpation method both bilaterally and simultaneously. Starting from the submental, submandibular region and going down, all the various cervical lymph node chains were evaluated including posterior auricular occipital and supraclavicular region.

Biopsy of Oral Squamous Cell Carcinoma Methodology: For patients with oral carcinoma, under general anesthesia, the lesion was excised along with radical neck dissection. For certain tuberculosis patients, under general anesthesia, the node biopsy was done. The specimen is collected in formalin, properly labelled and sent to the lab for histopathology examination. Sections of the node were done and stained with hematoxylin and eosin staining and studied under microscope.

Methodology for FNAB: For patients with inflammatory conditions and for certain patients with tuberculosis, FNAB of the node was done under local anesthesia using a 18 gauge needle and $10 \mathrm{cc}$ syringe. The needle was passed into the node after sterilization. Once the needle is passed, a negative pressure was created by aspirating the plunger. The nodal tissue was sucked into the needle and barrel. This was spread over the glass slide and smear was prepared. The glass slide was transferred to kouplin's jar containing ethyl alcohol for fixing. The slide is then stained with Hematoxylin and Eosin stain and studied under microscope.

Detailed Methodology of Using Color Doppler Sonography: All the patients were subjected to ultrasonography. For ultrasound examination of the cervical lymph nodes, the patients were made to lie supine on the examination couch with the shoulder supported by a pillow or triangular soft pad, and the neck hyperextended. The examination started with a transverse scan of the submental area. The transducer was then swept laterally to one side of the neck to the submandibular area, and the patient's head was turned away from the side under examination to allow free manipulation of the transducer. The submandibular area was examined with transverse scans along the inferior border of the mandibular body. As some of the submandibular nodes may reside behind the submandibular niche behind the body of the mandible, the transducer may need to be angled cranially to assess these lymph nodes.

The transducer was then swept laterally and superiorly along the angle of the mandible, and the mandibular ramus towards the pre-auricular region to examine the parotid region. Transverse and longitudinal scans were used to examine the parotid nodes. The internal jugular chain nodes are divided into upper, middle and lower cervical nodes. They were examined with the transducer scanning transversely from the tail of the parotid gland to the junction between the internal jugular vein and the subclavian vein, and along the common carotid artery and internal jugular vein.

The transducer was then moved laterally along the clavicle to the supraclavicular fossa, where the supraclavicular nodes were examined with transverse scans. A slight caudal angulation was required to avoid obscuring the lymph nodes by the clavicle. The posterior triangle nodes were examined with transverse scans from the mastoid tip to the acromion process along the imaginary course of spinal accessory nerve. Longitudinal scanning is occasionally used to assess the relationship between lymph nodes, especially when they may be matted.

The ultrasound probe was adequately adapted to the surface of the skin by applying a sufficient amount of gel particularly in the neck region bilaterally. Once the lymph node enlargement was identified, the ecogenicity, shape, borders, calcification or matting were assessed. The vascularity of the nodes was assessed by color doppler sonography.

Color doppler sonography evaluates the pattern of color doppler flow and the intranodal vascular resistance. Spectral doppler analysis was done in certain cases to evaluate the resistivity index and the pulsatility index. These features are used to differentiate between reactive and metastatic nodes.

\section{Results}

Six Cases of Squamous cell carcinoma were proved by biopsy. Out of the six cases, 3 cases were carcinoma of the alveolar mucosa, two cases were carcinoma of the tongue and one case was carcinoma of buccal mucosa. 
1) In carcinoma of alveolar mucosa of a 70-year-old male patient, clinically two submandibular nodes and one upper deep cervical node was palpated.

a. The two submandibular nodes were round, measuring $2.5 \mathrm{~cm}$, hard and fixed with tenderness. These two nodes on color doppler ultrasonography show the affected areas as round, sharp with regular outline. They are hypoechoic and heterogeneous with central necrosis and loss of echogenic hilus with no matting and calcification. Both nodes showed same characteristic features.

b. The upper deep cervical node was oval, measuring $1.5 \mathrm{~cm}$, hard, fixed and tender. The color doppler ultrasonography shows the node is round shape with regular border. The node is hypoechoic and heterogeneous with central necrosis and loss of echogenic hilus.

2) In the second case of carcinoma of left border of tongue, one left upper deep cervical node was round, measuring $1.5 \mathrm{~cm}$ in diameter, firm and tender. The color doppler ultrasonography showed the node to be ovoid with irregular border. The node was hypoechoic, homogenous with preservation of central fatty hilum.

3 ) In the third case of carcinoma of left buccal mucosa, the submandibular node was ovoid, hard, fixed and tender on palpation. The color doppler ultrasonography showed that the node was round with irregular border. The submandibular node was hypoechoic and heterogeneous with central necrosis and loss of echogenic hilus.

4) In the fourth case of carcinoma of the alveolus with pathological fracture, 3 nodes were enlarged (2 Submandibular and one Left upper deep cervical nodes)

a. The left submandibular node was round, $1 \mathrm{~cm}$ in diameter, fixed and tender on palpation. The color doppler ultrasonography showed that the node was round with irregular border. The submandibular nodes were hypoechoic and heterogenous with central necrosis and loss of echogenic hilus.

b. The right Submandibular node was round, $0.5 \mathrm{~cm}$ in diameter, firm, movable and non-tender on palpation. The color doppler ultrasonography showed that the node was ovoid in shape with regular border. The node was hypoechoic, homogenous with central fatty hilus.

c. The left upper deep cervical node was round, measuring $0.5 \mathrm{~cm}$ in diameter, firm and tender on palpation. The color doppler ultrasonography showed the node was round shape with regular border. The node was hypoechoic and heterogeneous with central necrosis and loss of echogenic hilus.

5) In the fifth case of carcinoma of tongue, the left submandibular node was enlarged. The left Submandibular node was round, $0.5 \mathrm{~cm}$ in diameter, fixed and tender on palpation. The colour doppler ultrasonography showed that the node was round in shape with irregular border. The submandibular nodes were hypoechoic and heterogeneous with central necrosis and loss of echogenic hilus.

6) In the sixth case of carcinoma of the alveolus with pathological fracture, 3 nodes were enlarged (left Submandibular and left upper deep cervical and left supraclavicular nodes)

a. The left submandibular node was oval, $2.5-5 \mathrm{~cm}$ in diameter, fixed and tender on palpation. The color doppler ultrasonography showed that the node was round in shape with regular border. The submandibular nodes were hypoechoic and heterogeneous with central necrosis and loss of echogenic hilus.

b. The left upper deep cervical node was round, fixed and tender on palpation. The color doppler ultrasonography showed the node was round shape with regular border. The node was hypoechoic and heterogeneous with central necrosis and loss of echogenic hilus.

c. The left supraclavicular node was round, fixed and tender on palpation. The color doppler ultrasonography showed the node was round shape with regular border. The node was hypoechoic and heterogeneous with central necrosis and loss of echogenic hilus.

In two cases of caseating Tuberculosis lymphadenitis, six nodes were enlarged.

1) In the first case,

a. One right upper deep cervical node was round, $1.5 \mathrm{~cm}$ in diameter, firm, movable and tender on palpation. The color doppler ultrasonography showed the node was ovoid shape with irregular border. The node was hypoechoic and heterogeneous with central necrosis and loss of echogenic hilus.

b. One right posterior auricular node was round, $0.5 \mathrm{~cm}$ in diameter, firm, movable and tender on palpation. The color doppler ultrasonography showed the node was ovoid shape with irregular border. The node was hypoechoic and heterogeneous with central necrosis and loss of echogenic hilus.

c. In right lower deep cervical region, the nodes were round, each $0.5 \mathrm{~cm}$ in diameter, firm, matted and non-tender on palpation. The color doppler ultrasonography showed the nodes were ovoid shape with irregular border. The nodes were hypoechoic and heterogeneous with central necrosis and loss of echogenic hilus.

2) In the second case of tuberculous lymphadenitis, one right upper deep cervical node was palpable, which was oval in shape, firm, matted and tender on palpation. The color doppler ultrasonography showed the node was ovoid shape with irregular border. The node was hypoechoic and heterogeneous with central necrosis and loss of echogenic hilus. Matted and 
specks of calcification of the nodes were also identified in the color doppler ultrasonography.

In a case of lymphoma, five nodes were enlarged. The right upper deep cervical node was round, firm, movable and tender on palpation. Three discrete right lower deep cervical nodes were round, discrete, firm, movable and non-tender on palpation. The left posterior auricular node was oval, firm and tender on palpation. The color doppler ultrasonography showed the nodes were ovoid shape with regular border. The nodes were hypoechoic and homogenous. There was loss of internal architecture with cystic appearance.

Six different inflammatory lymph nodes were considered for color doppler ultrasonography study.

In a case of pericoronitis, right submandibular node was enlarged. It was round, $1 \mathrm{~cm}$ in diameter, firm, movable and tender on palpation. In color doppler ultrasonography, the node was ovoid with irregular border, hypoechoic, homogenous with central fatty hilus preserved.

Two periodontal abscess cases, Submandibular node were enlarged.

In the first case, right submandibular node as ovoid, $1 \times 1.5 \mathrm{~cm}$ in diameter, firm, movable and tender on palpation. In color doppler ultrasonography study, the node was round with regular border, showing hypoechoic, homogenous with central fatty hilus preserved.

In the second case, left submandibular node was enlarged. The node was round, $1 \mathrm{~cm}$ in diameter, firm, movable and tender on palpation in color doppler ultrasonography study, the node was ovoid, irregular showing hypoechoic, homogenous with central fatty hilus preserved.

In sialolithiasis case, right submandibular node was enlarged. The node was round and $2 \mathrm{~cm}$ in diameter; it was firm, movable and tender on palpation. In color doppler ultrasonography study, the node was ovoid with regular border, hypoechoic, homogenous with central fatty hilus preserved.

In dentoalveolar abscess case, right submandibular node was enlarged, which was round, $1 \mathrm{~cm}$ in diameter, firm, movable and tender on palpation. In the colour doppler ultrasonography study, the node was ovoid, hypoechoic, homogenous with central fatty hilus preserved.

In periodontal abscess case, left submandibular node was enlarged. It was round, $1 \mathrm{~cm}$ in diameter, firm, movable and tender on palpation. In the color doppler ultrasonography study, the node was ovoid with irregular border, hypoechoic, homogenous with central fatty hilus preserved.

In an Impaction case, right submandibular and right upper deep cervical nodes were enlarged. Both the nodes were round and $1 \mathrm{~cm}$ in diameter. They were firm, movable and tender on palpation. In color doppler ultrasonography study, the right submandibular node was ovoid with irregular border, hypoechoic, homogenous with loss of echogenic hilus. The right upper deep cervical node was ovoid with irregular border, hypoechoic, and homogenous showing normal echogenic hilus.

Clinically, total number of cervicofacial lymph nodes evaluated in the study was 29 lymph nodes in 15 patients with various diseased states (oral cancer; tuberculosis; lymphoma and inflammatory conditions).

Using color doppler ultrasound, more than 70 lymph nodes were identified for the same patients. In patients with oral cancer (6 patients), clinically, only 12 lymph nodes were involved. Where as in color doppler ultrasound, 38 lymph node were identified.

Similarly for lymphoma, clinically only 5 lymph node were involved; but in color doppler ultrasound multiple nodal involvement were assessed bilaterally in the cervicofacial region.

Thus color doppler ultrasound plays a role in identifying the clinically missed lymph nodes, the prognosis of the diseased state.

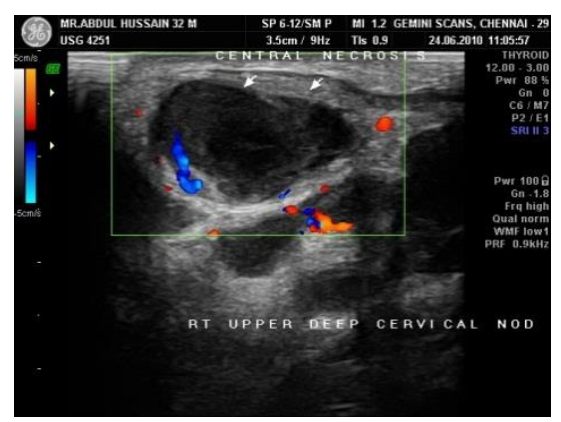

Fig. 1: Tuberculous lymph adenitis

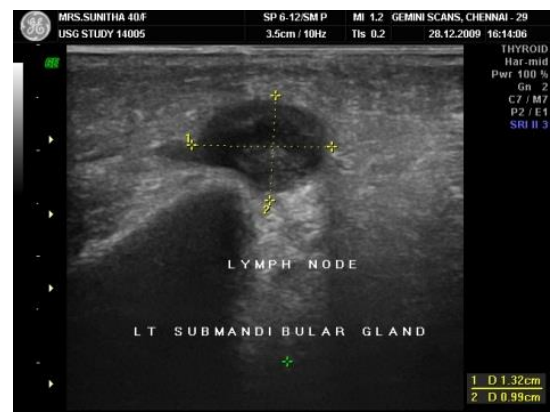

Fig. 2: CA buccal mucosa with nodal metastasis

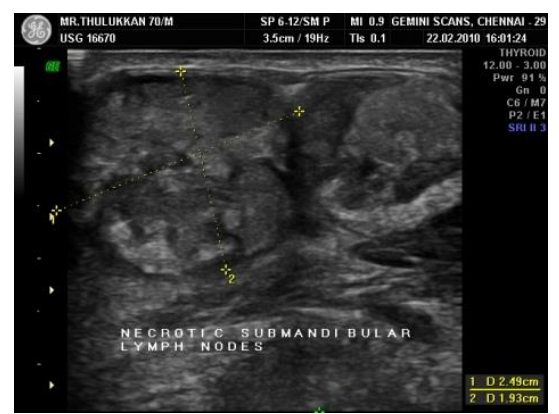

Fig. 3: CA alveolus with nodal metastasis 


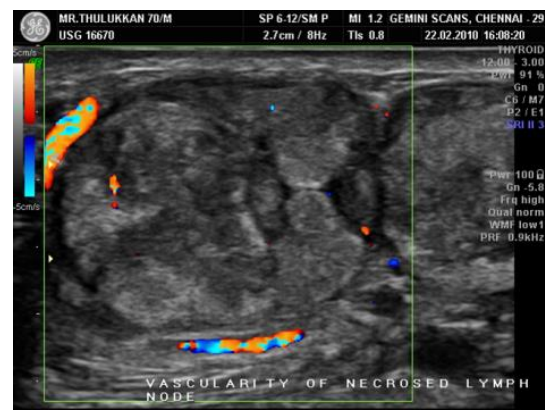

Fig. 4: CA alveolus with nodal metastasis

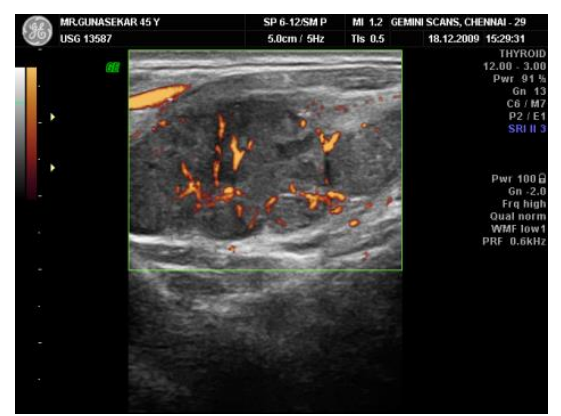

Fig. 5: CA buccal mucosa with metastasis

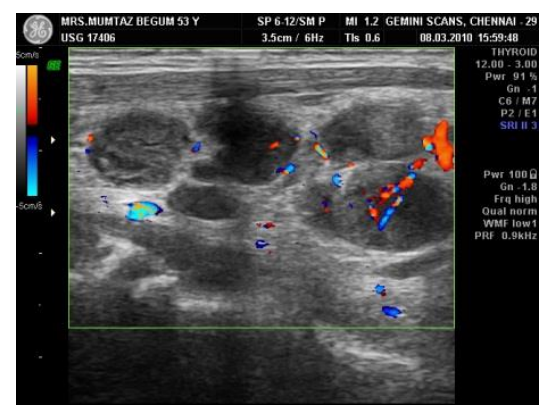

Fig. 6: CA alveolus with nodal metastasis

\section{Discussion}

Oral squamous cell carcinomas have got tendency to metastasize in the regional lymph nodes and distant lymph nodes. In the regional lymph nodes, carcinoma patients present with nodal enlargement that are movable, firm in consistency or fixed lymph nodes with stony hard consistency. Though oral squamous cell carcinoma can be confirmed by biopsy, in certain cases the biopsy cannot be conducted, and it requires an alternate method as in the case of bleeding disorder, leukemia, purpura and immunosuppressed state. ${ }^{2-4}$

The colour doppler ultrasonography is one of the recent imaging modalities which helps to diagnose lesions with particularly the vascularity of the lesion and the characteristic observation of carcinomatous and inflammatory nodes on CDUS was recorded. ${ }^{5}$

In six Squamous cell carcinoma cases, twelve nodes were seen clinically but totally thirty eight nodes have been detected in colour doppler ultrasonography. In each case, increased numbers of nodes have been recorded in ultrasonographical findings. This showed that many nodes that were not $\mathrm{t}$ detected clinically, were detected ultrasonographically. Thus the ultrasonographic observations with reference to a number of nodes, helps the operating surgeon to clear all the nodes in surgical resection of neck nodes. One interesting observation was that the submental lymph node that was not detected clinically, was detected by colour doppler ultrasonography. Thus, colour doppler ultrasonography is a proven method to detect submental node. $^{7}$

On comparing the clinical nodal enlargement with node echogenicity, it was found that out of twelve nodes, eight nodes have shown hypo echogenicity with heterogenous pattern and four nodes have shown Hypo echogenicity with homogenicity. Since all the nodes show only Hypo echogenicity, It can be determined that Hypo echogenicity is a characteristic of lymph nodes. ${ }^{8,9}$

With reference to the size and shape of the node, both clinically and colour doppler ultrasonographically, the node appears either ovoid or circular, clinically and ultrasonographically.

With reference to the ultrasonography observation of echogenic hilus, loss of echogenic hilus in a suspected node in colour doppler ultrasonography appears to be diagnostic feature of a metastatic node. ${ }^{10}$

With reference to pattern of colour doppler flow, in majority of carcinomatous lymph nodes, the flow was either peripheral or no flow. This variation shows that the vascular flow is a characteristic and typical feature in carcinomatous lymph node. Metastatic lymph node is not a vascularized one but consist of malignant cell infiltration into the nodal structure.

While studying the vascular resistance, that all the lymph nodes subjected to color doppler ultrasonography shows low vascular resistance. This indicated that the node has infiltrates of malignant cells without any vascular proliferations. ${ }^{11,12}$

An attempt has been made in this study, to record whether there are any matting and calcifications of regional lymph node enlargement in carcinoma cases as in tuberculosis cases. The recordings showed that metastatic lymph node involvement in squamous cell carcinoma do not have tendency to become matted or calcified. Since more and more malignant cells metastasize in node, the node merely enlarges and the full infiltration of malignant cells in the node causes a stony hard enlargement. The absence of calcification in the lymph node suggests that the malignant lymph nodes have any degenerations for calcification. ${ }^{13}$

The colour doppler ultrasonographic observations of lymph node in two tuberculous cases shows matting and so, matted lymph nodes occur only in tuberculosis and this suggests that when we suspect a node as a malignant lymph node and if it shows matted appearance in colour doppler ultrasonography, it cannot be diagnosed as malignant lymph node. ${ }^{14,15}$

With reference to internal architecture study in the lymph nodes of oral squamous cell carcinoma by colour doppler ultrasonography, it was found that all the 
lymph nodes show central necrosis except three nodes. Central necrosis is characteristic feature in metastatic carcinomatous lymph node. It was found that central necrosis of lymph node in tuberculosis is associated with matting and calcification. It was found that lymphoma shows no necrosis, but loss of internal architecture. In inflammatory lymph nodes, in cases like pericoronitis, peri odontal abscess, alveolar abscess, etc, the internal architecture of the node is preserved with central fatty hilus, hence central necrosis in the node is a feature in carcinomatous lymph node. ${ }^{16,17}$

The internal architecture of the node is destroyed by neoplastic infiltration. This will cause peripheral flow and no flow at all. the peripheral flow and no flow recorded in the pattern of colour doppler flow is due to internal necrosis as we recorded. No flow or absence of perfusion in lymph nodes is an important feature of colour doppler ultrasonography suggestive of malignant nature. ${ }^{18}$ The vascular resistance is uniformly low in many of the lymph nodes and this is due to the compression of blood vessels by fast proliferating malignant cells of the lymph node.

The present study of cervicofacial lymph node and colour doppler ultrasonography revealed many interesting features. Various changes have been seen in the nodes of oral squamous cell carcinoma. In carcinomatous lymph node, the shape of the node, border and loss of echogenic hilus with central necrosis of the node are all characteristic in the node of squamous cell carcinoma. If these features are present in colour doppler ultrasonography the node can be diagnosed as regional metastatic lymph nodes of oral squamous cell carcinoma. But the present study is only an attempt with six cases of carcinoma and more cases of Squamous cell carcinoma have to be studied for further confirmatory observations.

In conclusion, the utility of colour doppler ultrasonography is really beneficial in establishing the regional lymph node enlargement as metastatic lymph node enlargement in case of oral squamous cell carcinoma. However, the observation of present study cannot be concluded as the mandatory because the sample size is very less. With this study as the basis, further studies have to be conducted with a large number of oral squamous cell carcinoma for establishing the real facts and truth about the colour doppler ultrasonographic findings to diagnose lymph nodes in carcinomatous lesions.

\section{Conclusion}

Oral squamous cell carcinoma is more in India as $47 \%$ of total cancer. The etiology is not known and the definite treatment not designed. Any research study in this topic is certainly beneficial to find out a new point pertaining to oral cancer.

The present study is the study of color doppler ultrasonography of suspected metastatic regional lymph node in cervicofacial region of oral squamous cell carcinoma patient.

In the present study, 6 cases of oral squamous cell carcinoma and 7 cases of non-carcinomatous lesions were involved and there were subjected to colour doppler ultrasonography investigation.

Increased number of lymph node were recorded in colour doppler ultrasonography.

Sub mental lymphnode which cannot be detected clinically can be detected in color doppler ultrasonography.

In colour doppler ultrasonography, all nodes in oral squamous cell carcinoma are hypoechoic with Loss of echogenic hilus and the pattern of colour doppler flow peripheral or no flow with low or nil vascular resistance.

In conclusion, colour doppler ultrasonography observations lymph node in oral squamous cell carcinoma are showing encouraging positive points but this cannot be taken for a final conclusion as the number of case studied is less. However, the present observations could be a basis and further studies have to be done with more number of cases.

\section{References}

1. A.T. Ahuja, M Ying, S Y Ho, G. Antonio, Y.P.Lee, A.D. King \& K.T. Wong Ultrasound of Malignant Cervical Lymph nodes. Cancer Imaging(2008)8,48-56.

2. Alan D.L. Sihoe, Tak Wai Lee, Anil T. Ahuja and Anthony P.C. Yim; Patients with impalpable cervical lymph nodes? Should cervical ultrasonography be a routine staging investigation for lung cancer: Eur $\mathrm{J}$ Cardiothorac Surg 2004;25:486-91.

3. Anil T. Ahuja, Michael Ying; Sonographic evaluation of cervical lymph nodes. AJR:184; May 2005, 1691-169.

4. Ann D. King, Gary M. K. Tse, Anil T. Ahuja, Edmund H. Y. Yuen, Alexander C. Vlantis, Edward W. H. To, Andrew C. van Hasselt, Necrosis in Metastatic Neck Nodes: Diagnostic Accuracy of CT, MR Imaging, and US: Radiology 2004;230:720-6.

5. Chih-Hsiu Wu, Mow-Ming Hsu, Yih-Leong Chan, FonJou Hsieh, Vascular Pathology of Malignant Cervical Lymphadenopathy- Qualitative and Quantitative Assessment with Power Doppler Ultrasound; CANCER September 15, 1998, Volume 83;Number 6.

6. SEJ Connor and JFC, Imaging of malignant cervical lymphadenopathy. Dentomaxillofacial Radiology (2008)29;133-43.

7. S BDangore, SS Degwegar and RR Bhowate Evaluation of the efficacy of Color Doppler Ultrasound in diagnosis of Cervical lymphadenopathy. Dentomaxillofacial Radiology (2008)37,205-12.

8. W. Dennis Foley and Scott J.Ericson. Color Doppler flow imaging. IJR 156:3-13 Jan 1991.

9. Dong Gyu Na, Lim HK, Byun HS, Kim HD, Ko Yh Back JH. Differential diagnosis of cervical lymphadenopathy: Usefulness of color Doppler Sonography AJR May1997:168:1311-6.

10. EWH To, WM Tsang, J Cheng, E Lai, AT Ahuja;and M Ying; Is neck ultrasound necessary for early stage oral tongue carcinoma with clinically N0 neck? : Dentomaxillofacial Radiology (2003) 32, 156-9.

11. Francesco Dragon, Claudio Cartoni, Edoardo Pescarm, Flavia Chiarotti, Maria Puopolo, Enrico Orsi, M.D, 
Patrizia Pignoloni, Cinzia De Gregori, Franco Mandelli; The Role of High Resolution Pulsed and Color Doppler Ultrasound in the Differential Diagnosis of Benign and Malignant Lymphadenopathy- Results of Multivariate Analysis: CANCER June 1, 1999 / Volume 85 / Number 11, 2485-90.

12. Giovagnorio F, Galluzo M, Androli C, Decicco Vincenzo David- Color Doppler Sonography in the evaluation of Superficial Lymphomatous lymph nodes. J Ultrasound Med 2002;21;403-8.

13. Jorg D Moritz, Arwed Ludwig, Jorg W. oestmann. Contrast - enhanced color Doppler Sonography for evaluation of enlarged cervical lymph nodes in head and neck tumors. AJR:174 May2000;1279-84.

14. Madoka K Furukawa, Akira Kubota, Hideaki Hanamura, Yoshifumi Fujita, Masaki Furukawa. Diagnosis of Cervical lymph node metastasis of head and neck Squamous cell Carcinoma- Usefullness of power Doppler ultrasonography and elastography. Medix Suppl 2007;203.

15. Maria laura Ghirardelli, Vassili Jemos, Paolo G. Gobbi; Diagnostic approach to lymph node enlargement: Haematologica 1999; 84:242-7.

16. Mm Yun Choi1, Jun Woo Lee, Kyung Jae Jang; Distinction Between Benign and Malignant Causes of Cervical, Axillary, and Inguinal Lymphadenopathy: Value of Doppler Spectral Waveform Analysis: AJR 1995;165:981-4.

17. Misa Sumi, Masafumi Ohki, Takashi Nakamura; Comparison of Sonography and CT for Differentiating Benign from Malignant Cervical Lymph Nodes in Patients with Squamous Cell Carcinoma of the Head and Neck: AJR 2001;176:1019-24.

18. R.A. Mountfort, P. Attinsin. Doppler ultrasound examination of pathologically enlarged lymph nodes. British Journal of Radiol 1979,52;464-7. 